\title{
NextContact: Neighbor Discovery Mechanism for Opportunistic Networks
}

\author{
Prashant Kumar*, Naveen Chauhan, and Narottam Chand \\ Department of Computer Science \& Engineering \\ National Institute of Technology, Hamirpur, INDIA \\ prashantkumar32@gmail.com,naveen@nith.ac.in,nar@nith.ac.in
}

\begin{abstract}
Node discovery plays a vital role in opportunistic networks in order to efficient use of available resources as these networks suffered from intermittent connectivity. In opportunistic networks encounters are uncertain and it is difficult to determine the occurrence of next encounter. To make most out of the short contact duration it is essential that opportunistic networks must have an efficient node discovery procedure. In this article, we present a node discovery scheme called "NextContact". NextContact is based on the node movement. We investigate a formula to derive the probing interval to probe for the next encounter. Further we conduct simulation to validate the correctness of our proposed scheme.
\end{abstract}

Keywords: Opportunistic networks; neighbor discovery; probing interval; service discovery.

\section{Introduction}

By the tremendous evolution and growth in mobile communication, mobile phones become extremely popular and an integral part of our day-to-day life. Today's mobile phone are smartphones, which are equipped with powerful processors, rich communication capabilities that includes cellular telephony, Bluetooth and Wi-Fi. Beside from basic functioning (phone calls and SMS services etc.), today we depend on them for information acquisition, social networking, entertainment and much more. The capability and extensive use of smartphones make it feasible to disseminate the information in opportunistic network environment. With the advancement in communication technology and tremendous growth portable communication devices leads to the emergence of new peer-to-peer networks scenario like Opportunistic Networks (OppNets) [1]. OppNets is based on the instantaneous response of the devices. An OppNets grows from its seed. In the beginning set of nodes are employed together at the time of the initial OppNets deployment. The seed grows into a larger network by extending invitations to join the OppNets to other devices that are available [2]. These nodes are able to self-localize and self-configurable as per the network requirement. So OppNets is the platform, where various networking devices (as shown in Figure 1) with very diverse features of computing and communication, uses the environment to give the services to the user according their capabilities [3]. OppNets is formed by several nodes over a specific area. These nodes are the handheld devices carried by people and equipped with large memory, sensing capability, and high computational power with short-range radio transmission functionality and wirelessly connected to each other [4].

In OppNets stable end-to-end connectivity scarcely exist and data exchange is done when the nodes move in the transmission range of each-other. Nodes carry the data till next communication opportunity is found, thus data exchange in OppNets is based on store-carry-forward mechanism. The motivation to encourage the OppNets 
is to provide the connectivity and other network services thru natural calamities and in the remote areas where networks infrastructure is poor or not exists.
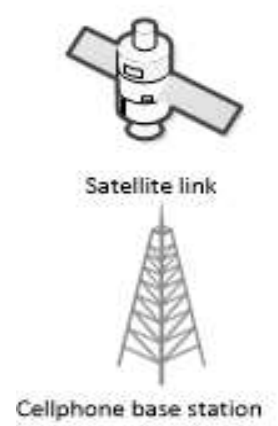

Cellphone base station

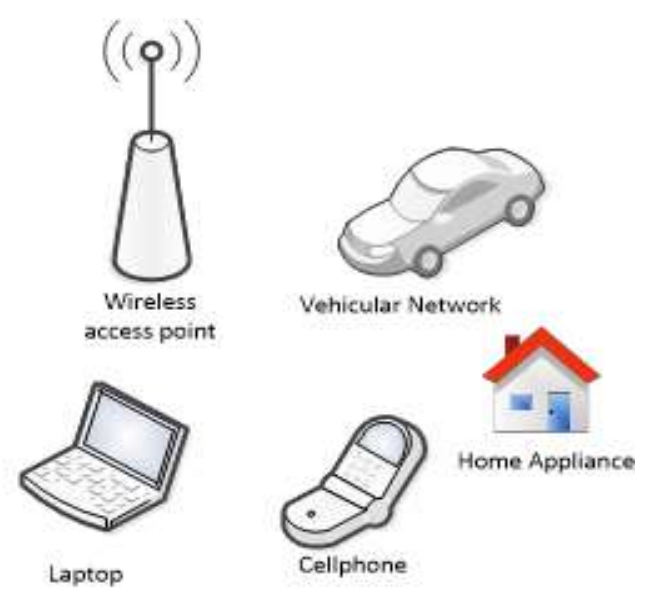

Figure 1. Devices used in OppNets

In the absence of permanent link, with low communication range and highly dynamic networks topology, it is essential that network must have to continuously listen to the environment to discover other nodes in the vicinity. Otherwise some contact opportunities might be missed. But continuous listening makes node discovery energy-intensive, as research shows that node discovery process requires as much energy as making a phone call [5]. Further neighbour discovery in Bluetooth is analysed by Trifunovic et al. [6]. Authors compare Bluetooth neighbour discovery with two innovative Wi-Fi based deviceto-device communications methods: Wi-Fi Direct [7] and WiFi-Opp [8]. Wi-Fi Direct and WiFi-Opp consumes two times and five times more energy than Bluetooth respectively. This indicates to the importance to probing interval i.e. how frequent they look into their neighbourhood for new device. As nodes are sparsely distributed in OppNets, so inter-connect time is generally larger than the contact duration. This leads to the fact that frequent probing also will consume the energy. Further if the probing interval is kept high, then some contact opportunities may be lost. Probing interval is a trade-off between contact opportunities and energy efficiency. As energy consumption is heavily depends upon probing interval, so it is desired to have some sort of ideal probing interval to make node discovery efficient and less energy consuming. In this article, we present a new equation to calculate the probing interval and proposed a new node discovery scheme called 'NextContact'. Our scheme is based on the node movement. We investigate a formula to derive the probing interval in OppNets.

Our contribution in this paper are as follows:

1. We propose an equation to calculate the probing interval.

2. Based on proposed equation, we propose a new neighbor discovery scheme, NextContact for OppNets.

3. We perform simulation to compare the performance of our proposed scheme with existing approaches.

Rest of the paper is organized as follows. Section 2 describe the related work. In Section 3, we discuss about the system model. The proposed algorithm, NextContact is described in Section 5. In Section 5, we present simulation result of the proposed algorithm. Finally, we conclude the paper in Section 6. 


\section{Related Works}

Node discovery algorithms in OppNets can be improved: by minimizing the power consumption in node discovery; by recognize the availability of nodes as well as also by learn when (or where) such node will be deemed available; by minimum the prior knowledge required; by shortening probing interval. However, if devices have some prior knowledge of encounters then this will be beneficial to reduce the power consumption.

Probing interval plays a vital role in node discover in OppNets. As OppNets consists short radio range device, probing interval becomes the bottleneck of performance of the discovery mechanism. If probing interval is kept too long, then short encounters being missed and contact loss probability will be high. Low probing interval will reduce the surely reduce the contact loss probability but it will require more energy. Figure 2 depicts the interface $\mathrm{ON}$ and OFF time. Let $\mathrm{T}$ is the system interval, then $T=T_{\text {on }}+T_{\text {off }}$. Let $\mathrm{D}_{\mathrm{c}}$ is the duty cycle, then $D_{c}=T_{\text {on }} / T$. Now the obvious question is which is better: low duty cycle with low contact loss or high duty cycle with high contact loss but saves the energy.

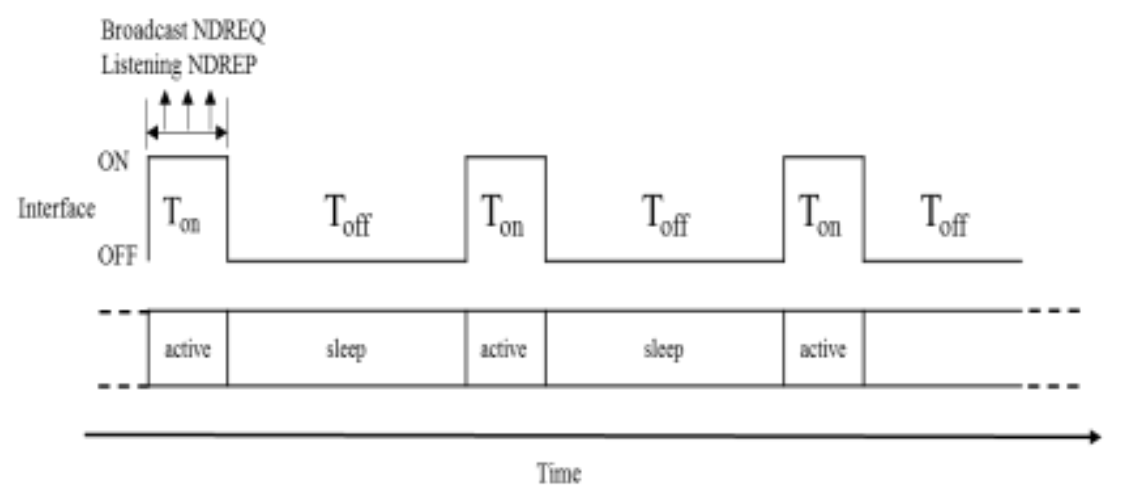

Figure 2. Duty Cycle Implementation in OppNets Nodes

Various authors proposed several schemes for node discovery in OppNets. Drula et al. [9] presents some observation on energy efficient node discovery in Bluetooth and proposed two protocols. These protocols are based on the fact that if whenever a contact opportunity is occurring then other contact opportunities are expected to occur. Pitkanen et al. [10] discuss about various factors that affect the discovery process in existing devices with their real precincts.

Izumikawa et al. propose an activation mechanism DWARF [11]. DWARF is based on NDREQ (Node Discovery Request) and NDRSP (Node Discovery Response). Authors claims that DWARF is 3.5 times more efficient than the fixedinterval scheme. DWARF requires no knowledge of previous encounters, but energy consumption is high in this protocol, as it requires continuous listening for signals. Wang et al. [5] proposed an adaptive contact probing scheme STAR, based on contact arrival process. STAR is an energy efficient protocol, but it fails to detect the short duration encounter that may lead to negative impact on network connectivity. Zhou et al. [12] propose a model for energy efficient contact probing in OppNets and establish relation among the detecting probability, radius of nodes and the speed of the nodes. Hess et al. [13] presents adaptive beaconing to reduce the scanning efforts, without missing many contacts.

Troël [14] proposed an equation to calculate the probing interval. According to this a device should be detected as neighbor when it comes within a threshold distance. However, Troel's equation assumes that the mobility of neighbor's 
inclines towards the center of the transmission range. This may not be the case in OppNets as human can travel along many paths. PISTONv1 and PISTONv2 are the two node movement based schemes proposed by Orlinski et al. [15], [16]. These schemes enable the mobile devices to alter the inter-probing time. These schemes improve over Troel's assumption, but consider only the absolute velocity of nodes while nodes may pause for while during the movement. So, it is better to consider the accumulate speed rather absolute speed.

\section{System Model}

This section describes about the system model followed in the work. The assumptions are summarized as follows:

1. OppNets are comprised of a group of $\mathrm{N}$ mobile nodes having basic computation and storage capacities.

2. The transmission range of all the nodes is assume to $\mathrm{R}$.

3. All nodes are moving with a homogeneous speed $\mathrm{V}$.

4. Moving speed is calculated by the devices itself by combining accelerometers readings [17].

5. The network is implemented over WLAN technology and nodes can communicate over Wi-Fi/Bluetooth.

Table 1 shows the notation used.

\section{Table 1. Notation Used}

\begin{tabular}{|l|l|}
\hline Notations & Meaning \\
\hline $\boldsymbol{R}$ & Range of nodes \\
\hline $\boldsymbol{V}$ & Moving speed of nodes \\
\hline $\boldsymbol{T}_{\text {pause }}$ & Pause time of nodes \\
\hline $\boldsymbol{D}$ & Distance travelled by the nodes \\
\hline$\overline{\boldsymbol{V}}$ & Accumulated speed of nodes \\
\hline$\overline{\boldsymbol{T}}_{\text {pause }}$ & Accumulated pause time of nodes \\
\hline$\overline{\boldsymbol{D}}$ & Accumulated distance travelled by the nodes \\
\hline $\boldsymbol{T}_{\text {Prob }}$ & Probing time of nodes \\
\hline $\boldsymbol{\Phi}$ & Constant \\
\hline NDREQ & Node discovery request \\
\hline NDREP & Node discovery reply \\
\hline
\end{tabular}

\section{Definitions:}

Contact: Two nodes $x$ and $y$ are said to be in contact with each other at time $t$ if and only if $\left|P_{x}(t)-P_{y}(t)\right| \leq R$, where $P_{x}(t)$ and $P_{y}(t)$ represent the geographical positions of node $x$ and $y$ at time $t$ respectively and $R$ is the transmission range of nodes, i.e. contact between the nodes is the instance when they first come within the transmission range of each other.

Contact duration: Contact duration is the difference between the times when they first come within the transmission range and when they first move outside from the transmission range of each other. Thus, the $k^{\text {th }}$ contact time between nodes $x$ and $y$, denoted as $t_{m}^{x y}(k)$ refers to the contact time since their $(k-1)^{\text {th }}$ leaving time, which is denoted as $t_{l}^{x y}(k-1)$ i.e. $t_{m}^{x y}(k)=\min \left\{t>t_{l}^{x y}(k-1),\left|P_{x}(t)-P_{y}(t)\right| \leq R\right\}$; and the $k^{\text {th }}$ leaving time between the nodes $x$ and $y$, which is denoted as $t_{l}^{x y}(s)$ is the leaving 
time since their $k^{\text {th }}$ contact time $t_{m}^{x y}(k)$, i.e. $t_{l}^{x y}(k)=\min \left\{t>t_{m}^{x y}(k),\left|P_{x}(t)-P_{y}(t)\right| \leq\right.$ $R\}$.

Probing time: Probing time is difference between the interface ON time and interface OFF time. $T_{\text {Prob }}=\left|T_{\text {on }}-T_{\text {off }}\right|$.

\section{Proposed Work}

In this section, we present our proposed scheme NextContact. The proposals in NextContact are based on nodes movement. To calculate the probing interval in OppNets, we proposed an equation. Our proposed equation is based on the idea that users can quickly cross one-another, as the communication range of OppNets devices are low. Hence, probing interval should be kept short. Figure 3 depicts the total time (T) spent by nodes in the range of each-other. We define probing interval, denoted by $T_{\text {prob }}(0<$ $T_{\text {prob }}<\infty$ ), as the time that gives shortest duration after which new neighbor discovery can be initiate. In order to detect the new neighbor $T_{\text {prob }}$ should be less than $\mathrm{T}$ ( $T_{\text {prob }}<$ $T$ ), otherwise contact opportunity will be missed.

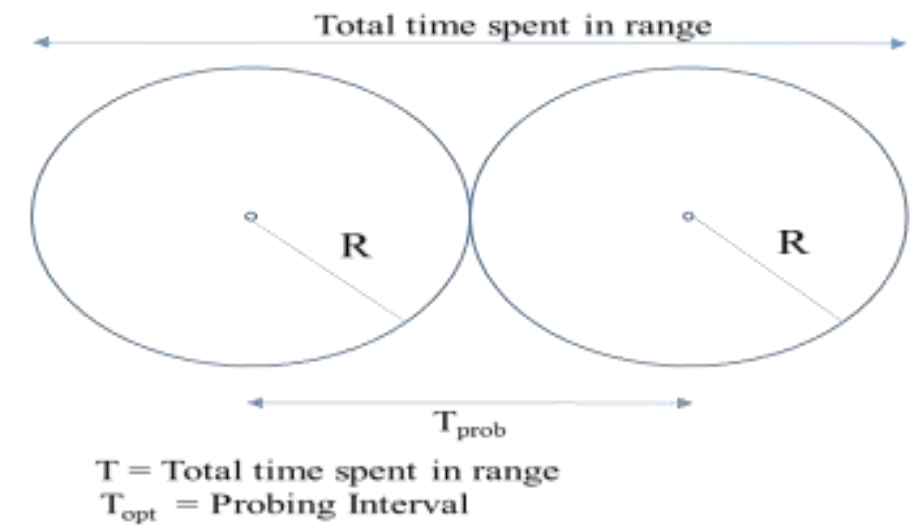

Figure 3. Time Spent by Two Nodes in the Range of Each-other

Equation (1) shows our proposed equation for calculating probing interval. We introduce a coefficient $\varphi(0<\varphi \leq 1)$. Equation (1) need the speed of node, $\mathrm{V}$, calculated in $\mathrm{m} / \mathrm{s}$ and transmission range of nodes, $\mathrm{R}$, in meters.

$$
T_{\text {prob }}=\frac{2 R}{\varphi V}
$$

As described earlier, in general, OppNets are formed by individuals (carrying a mobile device). In most of case these individuals are anonymous to each other. These individual (humans) communicates on spot if they found any communicating opportunity. As mobile devices are carried by the humans, so the mobility patterns in OppNets are very similar to human mobility. While visiting a place a person may stay at the destination for some time. Thus, pause time come in the role. When a person pause for a while, then it improves the probability of the detection. Further, speed calculated is also depends on the pause time as total time taken to cover a distance $\mathrm{d}$ is now $\mathrm{T}+\mathrm{T}_{\text {pause }}$. Hence the velocity is $V$ becomes accumulate velocity $\bar{V}$. The conclusion is that rather considering absolute speed of nodes, we must consider the absolute speed because during mobility nodes pause for some time. So, accumulated speed must be taken into account. Accumulated speed $\bar{V}$ is defined as

$$
\bar{V}=\frac{\bar{D}}{\bar{T}+\bar{T}_{\text {pause }}}
$$


Where $\bar{D}$ accumulative distance travelled, $\bar{T}$ is total time taken to travel $\bar{D}$ and $\bar{T}_{\text {pause }}$ is total pause time during $\bar{D}$.

Hence $T_{\text {prob }}$ is defined as:

$$
T_{\text {prob }}=\frac{2 R}{\varphi \bar{V}}
$$

The above equation produce the probing interval for OppNets environment. As mentioned earlier probing interval for OppNets device should be short as they reside for less time in each-others transmission range due to low communication range of devices. Equation (1) produce shorter probing interval as the value of range (R) decreases. Further for high mobility environment, the probing interval is also kept low as devices will cross each-other rapidly. This criterion is also fulfilled by proposed equation.

\subsection{Choosing the Value for Coefficient $\varphi$}

To show the variation in probing interval calculated by our proposed equation, we consider the speed $2 \mathrm{~m} / \mathrm{s}$ (like a person walking leisurely) and communication range of device is $10 \mathrm{~m}$. At $\varphi=1 ; T_{\text {opt }}=10 \mathrm{~s}$ that means after every $10 \mathrm{~s}$ a new neighbor discover will starts. However, to conserve the energy value of $\varphi$ is decreased as per the application scenario, like in situation where detection of all new opportunities is not required. The effects of speed variation can be seen. If the speed of users increases the probing interval decrease. Figure 4 shows the effect of speed on the probing interval at different values of $\varphi$.

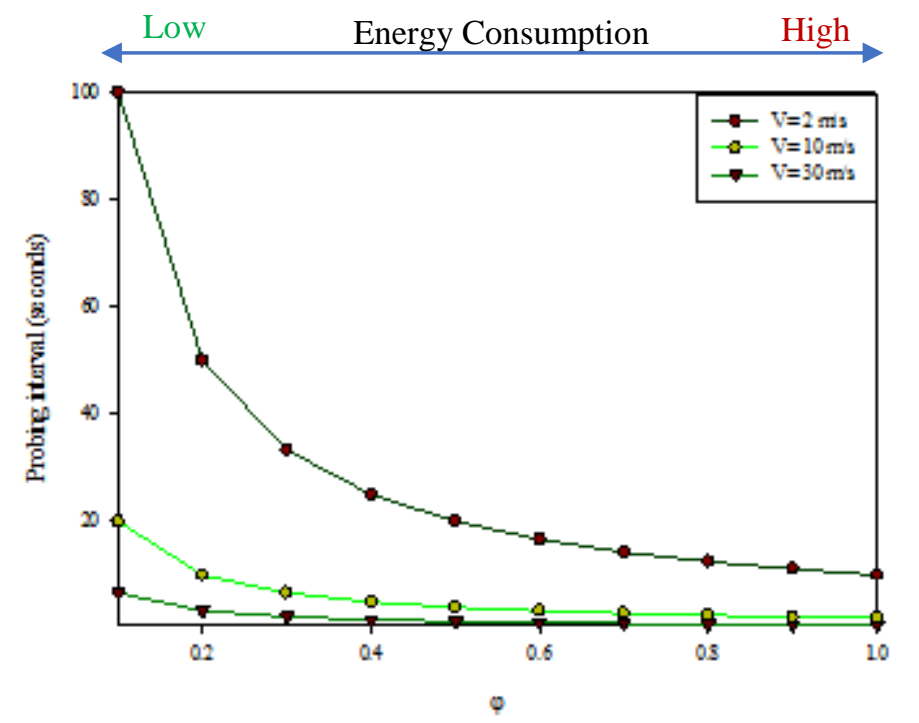

Figure 4. Probing Interval Produces under Different Values of $\varphi$ with varying Speed at Communication Range $R=10 \mathrm{~m}$.

To show the effect of device communication range, we consider the range of devices $10 \mathrm{~m}, 20 \mathrm{~m}$ and $30 \mathrm{~m}$. To probing interval is calculated at these values, speed of devices is kept fix at $10 \mathrm{~m} / \mathrm{s}$. The proposed equation produce the shorter probing interval, which is required to detect the maximum contact opportunities. These variations in the probing interval with different values for $\varphi$ is shown in Figure 5. 


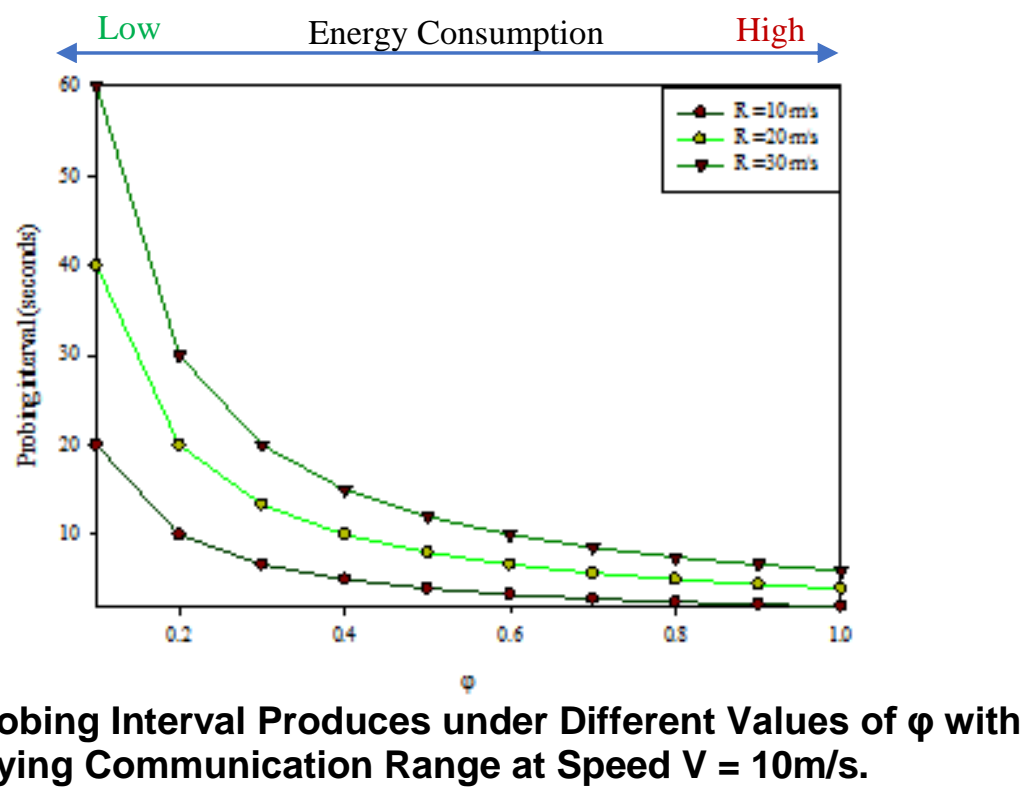

\subsection{Working of NextContact}

This section describes about the neighbor discovery process. Our neighbor discovery process is based on NDREQ (Node Discovery Request) and NDREP (Node Discovery Reply). Whenever a node turn on its interface, will look for NDREQ. If NDREQ is received, then pairing is initialized with the $N D R E Q$ sender by sending NDREP. If not, then node will have checked when last neighbor discovery process was initiated. If current time (CurrentTime) is greater than last search time (LastSearch) and probing interval (ProbingInterval), the node will initiate the new neighbor discovery process. For this, node will broadcast $N D R E Q$ and set the value of $T_{\text {prob }}$ as per equation (1). If $N D R E P$ is received the pairing will initialized with the responder and current time is set as the last search time and the interface is turn off to save the energy.

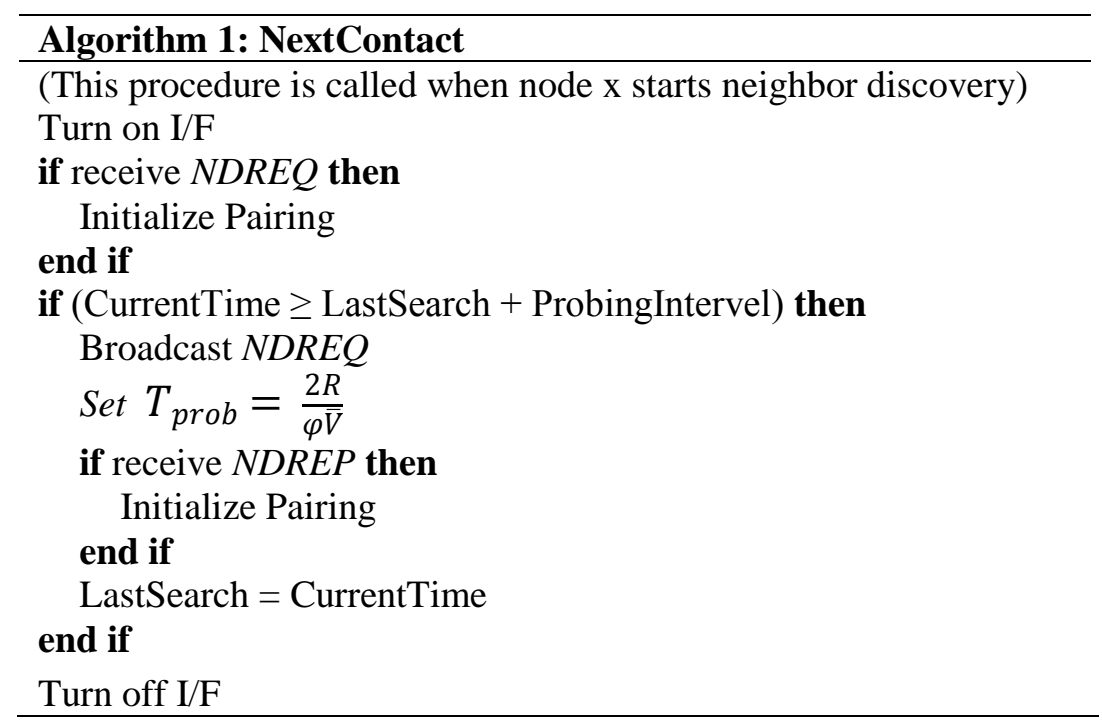




\section{Simulation \& Results}

In this section, we present the simulation results for our proposed neighbor discovery mechanism, NextContact. We use the ONE simulator [18] to evaluate our proposed method. Our simulation model is similar to the model used in [16]. We set speed $2 \mathrm{~m} / \mathrm{s}$ for slow movement and $30 \mathrm{~m} / \mathrm{s}$ for fast movement. Transmission range is $10 \mathrm{~m}$ and simulation area is $500 \mathrm{~m} \times 500 \mathrm{~m}$. Table 2 summarizes the simulation parameters. NextContact is compared with PISTONv2 [16]. The performance metric used is percentage of neighbor discovered and energy required per neighbor discovery. We also evaluate NextContact under the delivery ratio with Spray and Focus [19] routing protocol.

Table 2. Simulation Parameters

\begin{tabular}{|l|l|l|}
\hline Notations & Parameter & Default Value \\
\hline Simulation area & $500 \mathrm{~m} \times 500 \mathrm{~m}$ & \\
\hline Number of nodes & $50 \sim 150$ & 80 \\
\hline Transmission range & $10 \mathrm{~m} \sim 30 \mathrm{~m}$ & $10 \mathrm{~m}$ \\
\hline Transmission rate & $3 \mathrm{Mbps}$ & \\
\hline Movement speed & $2 \mathrm{~m} / \mathrm{s} \sim 30 \mathrm{~m} / \mathrm{s}$ & $10 \mathrm{~m} / \mathrm{s}$ \\
\hline Mobility model & Random Way Point & \\
\hline Message generation rate & $\begin{array}{l}4 \sim 5 \text { messages per } \\
\text { min }\end{array}$ & \\
\hline Size of messages & $500 \mathrm{~KB} \sim 1 \mathrm{MB}$ & \\
\hline
\end{tabular}

\subsection{Effect of $\varphi$ on Percentage of Neighbor Discovered}

In Figure 6 we plot the number of neighbor discovered with the different values for $\varphi$. These results are taken while nodes move at slow speed. NextContact produces better probing intervals hence perform better than PISTONv2. Similarly, the results shown in Figure 7 are taken while nodes move at high speed. Our proposed method performs better in this case also. It can be seen in the both scenarios that most of the encounters detected when values of coefficient $\varphi$ is cross 0.4 . When values of coefficient $\varphi$ is crossed over 0.6 , increase in the percentage of neighbor discovered is almost negligible. Hence one conclusion can be drawn that ideal value of $\varphi$ is lies between 0.4 to 0.6 .

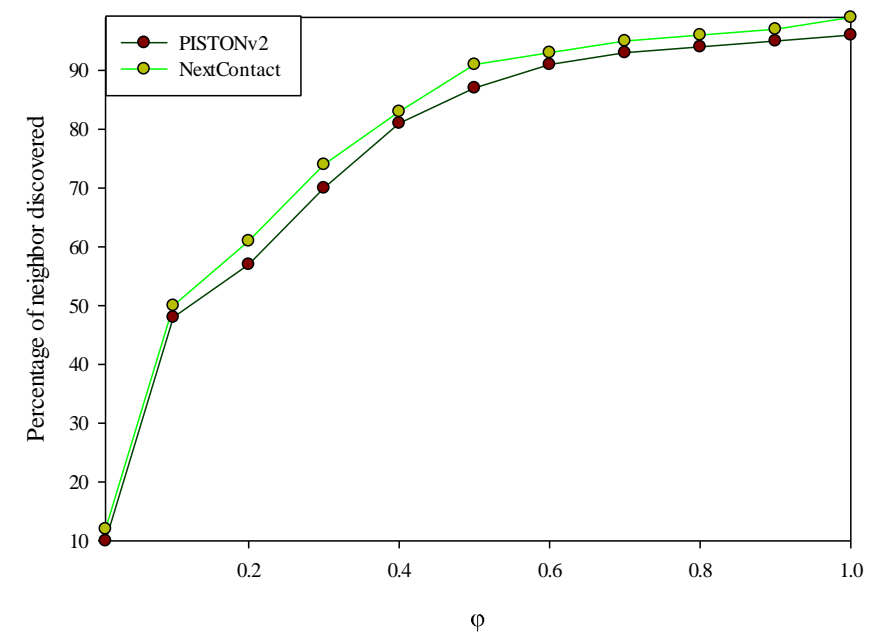

Figure 6. Percentage of Neighbor Discovered with Different Values of $\varphi$ under Slow Movement 


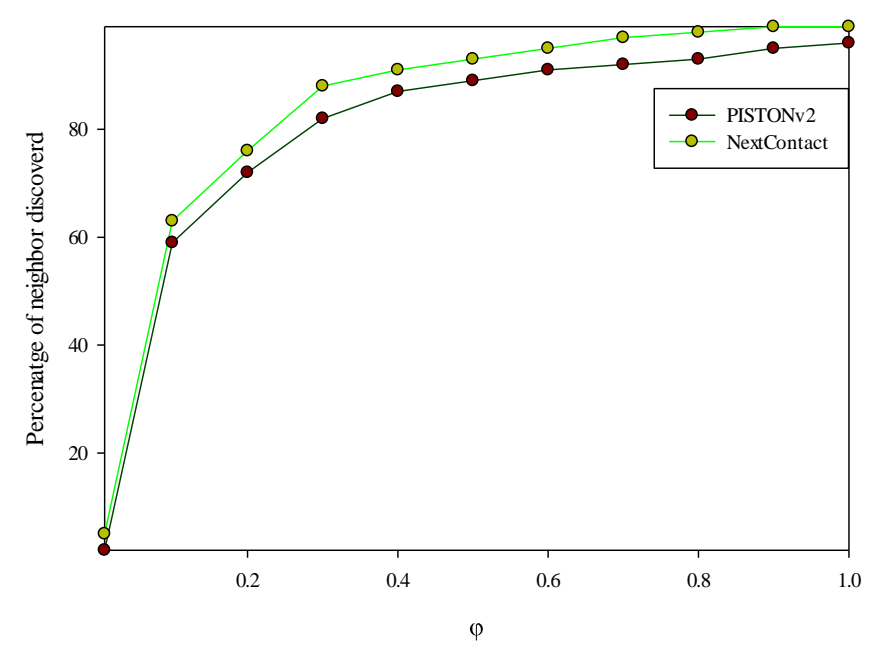

Figure 7. Percentage of Neighbor Discovered with Different Values of $\varphi$ under Fast Movement

\subsection{Effect of $\varphi$ on Energy Consumption}

In Figure 8 we plot the energy required per neighbor discovery with the variation in the different values of $\varphi$. Neighbor discovery energy includes the energy required in broadcasting a NDREQ packets, responding to NDREP packets. As seen in Figure 8, as the values of $\varphi$ is increases the energy consumption is increases. NextContact is an energy adaptive protocol and according to application scenario energy consumption can be controlled by adjusting the values of $\varphi$. Further, NextContact is more energy efficient than PISTONv2. This is due to the fact that NextContact produces 4 times probing interval than PISTONv2. Hence, in PISTONv2 more energy is waste in interface activation and deactivation process. Study conducted by Wang et al. [5] shows that making the device to be discoverable is a costly affair.

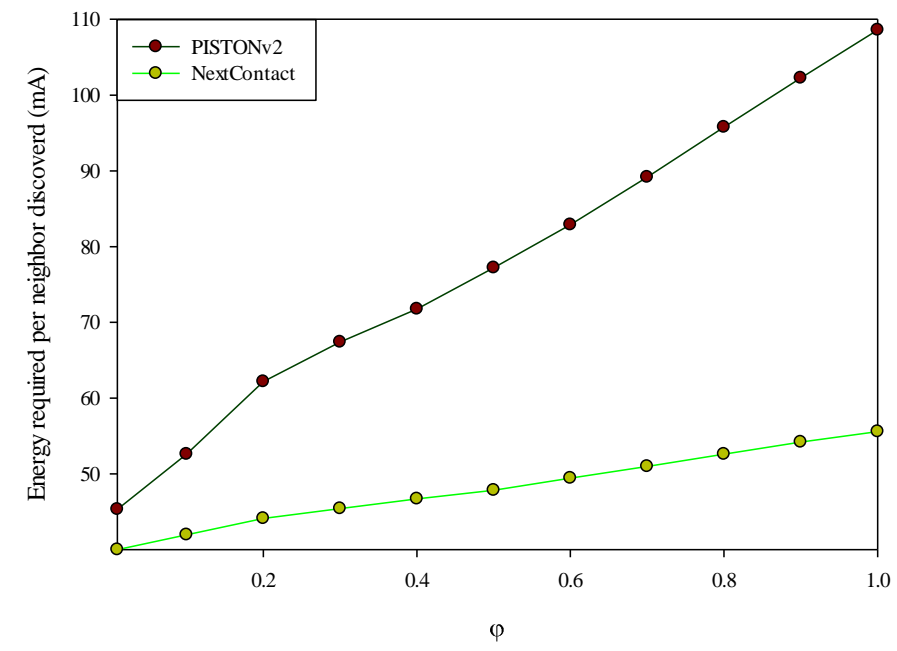

Figure 8. Effect of $\varphi$ on Energy Required per Neighbor Discovered

\subsection{Effect of Probing Interval on Delivery Ratio}

To show the effect of the probing interval we adapt spray and focus [19] as routing protocol. To study the effect, first we plot delivery ratio as function of $\varphi$. Figure 9 shows delivery ratio with the variation in the values of $\varphi$. It can be observed from the plot that NextContact exhibits better delivery ratio than PISTONv2. This is due to the fact that 
in a given period of time NextContact discover more neighbors which turns into more opportunities of data carriers hence the delivery ratio increase. Similarly, in Figure 10 we plot delivery ratio as function of number of nodes. It can be seen from the plot as the number of nodes increases delivery ratio increase. NextContact performs better than PISTONv2 in this case too.

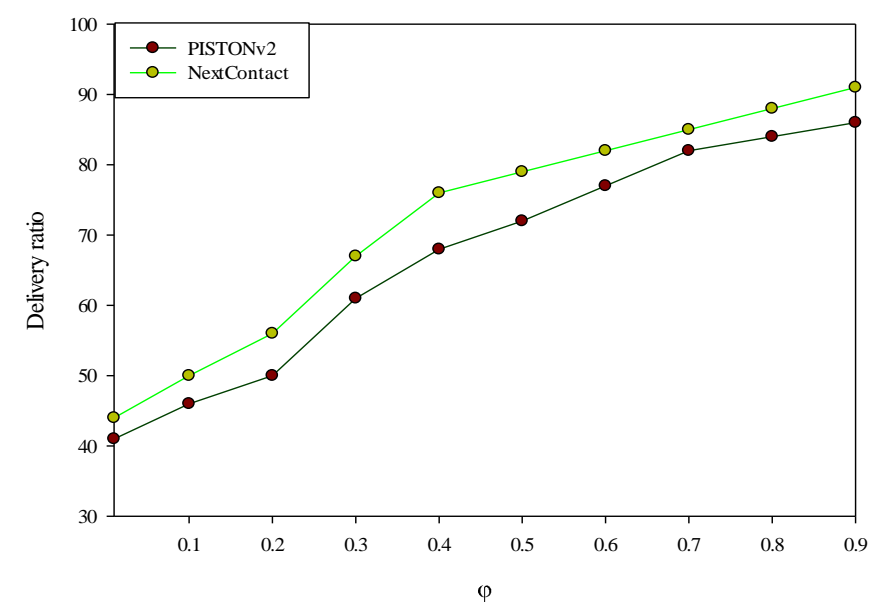

Figure 9. Effect of $\varphi$ on Delivery Ratio

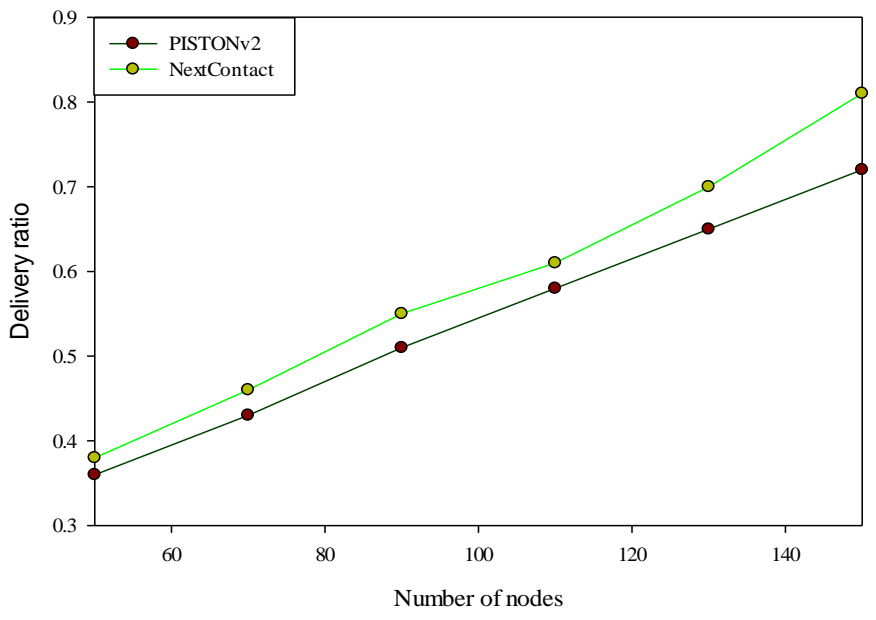

Figure 10. Effect of Number of Nodes on Delivery Ratio

\section{Conclusion}

In this paper we discuss about the efficient node discovery in opportunistic networks and investigate a new equation to calculate the probing interval by considering the node movement. Our equation produce adaptive probing interval with respect to changes in the speed and the transmission range of the nodes. Further our proposed equation is adaptive for the application scenarios too. By choosing the value of coefficient $\varphi$, energy consumption can be controlled, for example, in a scenario where all neighbors are not necessary to be discovered, the value of $\varphi$ can put low. Based on proposed equation we also proposed a new neighbor discovery process, NextContact for the opportunistic networks. We perform simulation to test our proposals and results show that proposed mechanism NextContact perform well under different networks scenarios. 


\section{References}

[1] C.-M. Huang, K. Lan and C.-Z. Tsai, "A Survey of Opportunistic Networks”, 22nd International Conference on Advanced Information Networking and Applications - Workshops, (2008).

[2] L. Lilien, Z. H. Kamal, V. Bhuse and A. Gupta, "Opportunistic Networks : The Concept and Research Challenges in Privacy and Security", Proc WSPWN, vol. 140, (2006), pp. 134-147.

[3] M. Conti and S. Giordano, "Mobile ad hoc networking: Milestones, challenges, and new research directions", IEEE Commun. Mag., vol. 52, no. 1, (2014), pp. 85-96.

[4] A. Chaintreau, P. Hui, J. Crowcroft, C. Diot, R. Gass and J. Scott, "Impact of human mobility on opportunistic forwarding algorithms”, IEEE Trans. Mob. Comput., vol. 6, no. 6, (2007), pp. 606-620.

[5] W. Wang, M. Motani and V. Srinivasan, "Opportunistic Energy-Efficient Contact Probing in DelayTolerant Applications”, vol. 17, no. 5, (2009), pp. 1592-1605.

[6] S. Trifunovic, A. Picu, T. Hossmann and K. A. Hummel, "Slicing the Battery Pie: Fair and Efficient Energy Usage in Device-to-Device Communication via Role Switching", Proc. 8th ACM MobiCom Work. Challenged networks - CHANTS, (2013).

[7] "WiFi-Direct", [Online]. Available: http://www.wi-fi.org/. [Accessed: 30-Apr-2016]

[8] S. Trifunovic, B. Distl, D. Schatzmann and F. Legendre, "WiFi-Opp: Ad-Hoc-less Opportunistic Networking", Proceedings of the 6th ACM workshop on Challenged networks, (2011).

[9] C. Drula, C. Amza, F. Rousseau and A. Duda, "Adaptive energy conserving algorithms for neighbor discovery in opportunistic bluetooth networks", IEEE J. Sel. Areas Commun., vol. 25, no. 1, (2007), pp. 96-107.

[10] M. Pitkanen, T. Karkkainen and J. Ott, "Mobility and Service Discovery in Opportunistic Networks", EEE International Conference on Pervasive Computing and Communications Workshops, (2012).

[11] H. Izumikawa, M. Pitkanen, J. Ott, A. Timm-giel and C. Bormann, "Energy-Efficient Adaptive Interface Activation for Delay/Disruption Tolerant Networks", ICACT, (2010), pp. 645-650.

[12] H. Zhou, J. Chen, H. Zheng and J. Wu, "Energy Efficiency and Contact Opportunities Trade-off in Opportunistic Mobile Networks", IEEE Trans. Veh. Technol., vol. 65, no. 5, (2016), pp. 3723-3734.

[13] A. Hess, E. Hyytia and J. Ott, "Efficient neighbor discovery in mobile opportunistic networking using mobility awareness", Sixth International Conference on Communication Systems and Networks, (2014).

[14] A. Troël, "Prise en compte de la mobilité dans les interactions de proximité entre terminaux á profils hétérogénes", Université de Rennes I, (2004).

[15] M. Orlinski and N. Filer, "Movement Speed Based Inter-Probe Times for Neighbour Discovery in Mobile Ad-hoc Networks", pp. 1-16.

[16] M. Orlinski and N. Filer, "Ad Hoc Networks Neighbour discovery in opportunistic networks", Ad Hoc Networks, vol. 25, (2015), pp. 383-392.

[17] L. Bedogni, M. Di Felice and L. Bononi, "By train or by car? Detecting the user's motion type through smartphone sensors data", IFIP Wirel. Days, (2012), pp. 1-6.

[18] A. Keranen, T. Karkkainen and J. Ott, "Simulating Mobility and DTNs with the ONE", J. Commun., vol. 5, no. 2, (2010), pp. 92-105.

[19] T. Spyropoulos, K. Psounis and C. S. Raghavendra, "Spray and Focus : Efficient Mobility-Assisted Routing for Heterogeneous and", IEEE International Conference on Pervasive Computing and Communications Workshops, (2007). 
International Journal of Grid and Distributed Computing

Vol. 10, No. 1 (2017) 